\title{
La parálisis cerebral como una condición dinámica del cerebro: un estudio secuencial del desarrollo de niños hasta los 6 años de edad*
}

\author{
Cerebral Palsy as a Dynamic Condition of the Brain: A Sequential \\ Study of the Development of Children up to 6 Years of Age
}

Recibido: febrero 8 de 2010 | Revisado: octubre 5 de 2010 | Aceptado: marzo 3 de 2012

\author{
ENRIQUE MORALEDA BARRENO ** \\ MODESTO JESÚS ROMERO LÓPEZ *** \\ María José CAYETANO MENÉNDEZ ***** \\ Universidad de Huelva, España
}

SICI: 1657-9267(201303)12:1<119:PCCDCE>2.0.TX;2-Y

Para citar este artículo: Moraleda, E., Romero, M. J. \& Cayetano, M. J. (2013). La parálisis cerebral como una condición dinámica del cerebro: un estudio secuencial del desarrollo de niños hasta los 6 años de edad. Universitas Psychologica, 12(1), 119-127.

* Trabajo financiado por el Servicio de Neuropsicología y Rehabilitación (SEREN) y realizado gracias al convenio específico de colaboración (2008. M06.17-4) entre el Hospital Juan Ramón Jiménez y la Universidad de Huelva, España.

** Doctor en Psicología. Departamento de Psicología Clínica, Experimental y Social, Campus de «El Carmen» Avda. Fuerzas Armadas, s/n. 21071, Huelva, España. Neuropsicólogo del Servicio de Evaluación y Rehabilitación Neuropsicológica SEREN. Tel: +349592826493.E-mail: enrique.moraleda@dpsi. uhu.es

**** Doctor en Psicología. Departamento de Psicología Clínica, Experimental y Social, Universidad de Huelva. Campus de «El Carmen» Avda. Fuerzas Armadas, s/n. 21071, Huelva, España. Neuropsicólogo del Servicio de Evaluación y Rehabilitación Neuropsicológica SEREN. Tel: +34 9592826493. E-mail: modesto.romero@dpsi.uhu.es

****** Departamento de Psicología Clínica, Experimental y Social. Campus de «El Carmen» Avda. Fuerzas Armadas, s/n. 21071, Huelva, España. Psicóloga y Logopeda del Servicio de Evaluación y Rehabilitación Neuropsicológica SEREN. Tel: +349592826493. E-mail: mjose.cayetano@dpces.uhu.es

\section{RES UMEN}

La parálisis cerebral es un trastorno motor debido a inmadurez cerebral con numerosos déficits asociados, incluidos los adaptativos, sociales, motores, cognitivos y de la comunicación, y con gran impacto en el desarrollo. El objetivo del presente estudio fue estudiar el desarrollo durante un año de niños con parálisis cerebral de uno a seis años. El método empleado fue un diseño ex post facto evolutivo secuencial. Se utilizó la prueba de screening del Inventario de Desarrollo de Battelle en un pretest y en un postest transcurrido un año. La muestra estuvo compuesta por 41 niños de entre 1 y 6 años de edad con diagnóstico de parálisis cerebral infantil. Los niños mostraban puntuaciones en el postest significativamente superiores en todas las áreas estudiadas. Se observó una mejora generalizada en las capacidades de la mayoría de los sujetos. Se discuten las posibles influencias que contribuyen a estas mejoras en los cocientes de desarrollo en la obtención de estos resultados.

Palabras clave autores

Parálisis cerebral, escalas de desarrollo, diagnóstico temprano, educación especial. Palabras clave descriptores

Trastornos del desarrollo, neuropsicología, España.
A B S T R A C T
Cerebral palsy is a motor disorder due to brain immaturity associated with deficit including adaptive deficits, social, engines, cognitive and commu- nication and with great impact on development. The aim was to study the development during a year of children with cerebral palsy from one to six years. The method was a sequential evolutionary ex post facto design. We utilized the screening test of the Battelle Developmental Inventory in a pre-test and a post-test after one year. The sample consisted of 41 children aged between 1 and 6 years of age diagnosed with cerebral palsy. Children in the posttest scores showed significantly higher in all areas studied. There has been an overall improvement in the capabilities of most subjects. Are discussed the possible influences contributing to these improvements in the ratios of development to obtain these results.
Key words authors
Cerebral palsy, developmental scales, early diagnosis, special education.
Key words plus
Development Disorder, Neuropsychology, Spain. 


\section{Introducción}

Según su definición clásica, la parálisis cerebral infantil (PCI) es un trastorno de los movimientos y la postura debido a un defecto de inmadurez cerebral (Bax, 1964). Este trastorno se ha entendido como una condición estática y no progresiva del cerebro en la infancia; sin embargo, según algunos autores, es muy común que se produzcan cambios en las manifestaciones motoras con el continuo desarrollo del cerebro, por lo que debería considerarse una condición dinámica (Bax \& Brown, 2004). El término parálisis cerebral es un concepto vago e impreciso que no puede considerarse como una entidad nosológica o enfermedad, sino como una agrupación sindrómica que tiene utilidad en la práctica clínica (Nieto \& Candau, 1995). Las causas pueden ser muy diversas, y entre ellas se encuentran las prenatales (generalmente de origen desconocido), las complicaciones en el nacimiento (como asfixia), el bajo peso al nacer (inferior a 2.500 gramos y sobre todo inferior a 1.500 gramos), la prematuridad, el crecimiento intrauterino retardado, las hemorragias intracraneales, los traumatismos craneoencefálicos, la meningitis bacteriana, la encefalitis vírica y la hiperbilirubinemia (Krigger, 2006). Alrededor de dos tercios de los casos presentan anormalidades en las técnicas de neuroimagen que incluyen infartos cerebrales focales, malformaciones cerebrales y leucomalacia periventricular (O'Shea, 2008).

Los niños con diagnóstico de parálisis cerebral suelen sufrir importantes retrasos en el desarrollo antes de los dos años, evidenciadas en las escalas de desarrollo (Van Handel, Swaab, de Vries \& Jongmans, 2007). La mayoría de ellos, no solo sufren trastornos motores, sino también otros déficits que pueden tener un gran impacto en su desarrollo (Liptak \& Accardo, 2004), entre los que destacan la epilepsia, los trastornos del habla y las alteraciones visuales y auditivas. La presencia de estas complicaciones se ha relacionado con la disminución de las capacidades cognitivas (Pueyo \& Vendrell-Gómez, 2002). La incidencia de la epilepsia se encuentra entre el $28 \%$ y el $42 \%$ (Zafeiriou, Kontopoulos \& Tsikoulas, 1999) y algunos autores consideran que su presencia está directamente relacionada con los síntomas cognitivos (Cioni et al., 1999; Vargha-Khadem, Isaacs, Van Der Werf, Robb \& Wilson, 1992). Los déficits cognitivos se producen entre un $40 \%$ y un $65 \%$ de los casos (Beckung \& Hagberg, 2002; Surman, Newdick \& Johnson, 2003), los trastornos del habla afectan aproximadamente al $56 \%$ de los niños con parálisis cerebral mientras que el $40 \%$ sufre alteraciones visuales y entre el 10 y el $16 \%$, alteraciones auditivas (Zafeiriou et al., 1999). Los niños con parálisis cerebral corren riesgo de sufrir aislamiento social y rechazo de los iguales, lo cual, junto a la falta de capacidades funcionales y de independencia personal, puede causar problemas adicionales de salud mental (Manuel, Naughton, Balkrishnan, Paterson-Smith \& Koman, 2003).

El $80 \%$ de los niños con esta patología presenta algún tipo de trastorno en la adquisición del lenguaje, que varía entre dificultades leves y alteraciones graves y que puede afectar a diferentes componentes del lenguaje sin que pueda establecerse un patrón general en cuanto a tales dificultades (Puyuelo, 2001). El lenguaje se ve perturbado tanto desde el punto de vista motor, que incluye los reflejos orales, respiración, fonación, articulación y prosodia, como desde el lingüístico, afectando a la fonética, morfosintaxis, léxico y pragmática (Puyuelo, 1998).

La incidencia del retraso mental se sitúa entre el $30 \%$ y el $70 \%$, siendo grave entre el $14 \%$ y el 59 \% (Hagberg, Hagberg \& Zetterström, 1989) y su presencia se ha relacionado con la existencia de diferentes lesiones cerebrales, tales como alteraciones en la sustancia blanca, afectación córtico-subcortical, dilatación ventricular y lesión cortical (Humphreys, Whiting \& Pham, 2000; Krägeloh-Mann et al., 1995). Algunos autores han propuesto que existe relación entre los diferentes tipos de PCI (hemiplejia izquierda o derecha, pacientes espásticos o atetósicos) y el CI (Pueyo \& Vendrell-Gómez, 2002), pero la mayoría de las investigaciones no han encontrado esta relación. Distintos estudios consideran que las dificultades cognitivas se acrecientan con la edad (Puyuelo, 2001) de manera que algunos niños que mostra- 
ban un rendimiento cognitivo adecuado en la primera infancia, comienzan a presentar trastornos del aprendizaje en la edad escolar.

Las actuales definiciones de retraso mental hacen hincapié en la disfunción de múltiples áreas de las habilidades cognitivas, lingüísticas, motoras y sociales (Damman \& Levinton, 1997) y no solo en el cociente intelectual bajo. Los niños con parálisis cerebral y deterioro cognitivo reducen sus niveles de participación en numerosos ámbitos de la vida, tales como las actividades recreativas, el autocuidado, la comunicación, la vida familiar, la movilidad, las responsabilidades, las relaciones personales y sociales o el colegio (Parkes, $\mathrm{McCu}$ llough \& Madden, 2010).

Según la American Psyquiatric Association (1994), se considera que una persona sufre retraso mental cuando su CI es inferior a 70; sin embargo, entre el 15 y el $20 \%$ de los niños con parálisis cerebral tienen grandes dificultades para responder a las pruebas de inteligencia, de modo que a menudo a los niños con discapacidad clínica grave se les atribuye una deficiencia mental exclusivamente o en parte sobre la base de la experiencia clínica (Andersen et al., 2008; Himmelmann, Hagberg, Wiklund, Eek \& Uvebrant, 2007). Para evaluar el nivel de desarrollo en niños pequeños suelen utilizarse escalas de desarrollo como las Escalas Bayley de Desarrollo Infantil o el Inventario de Desarrollo de Battelle, que evalúan las distintas áreas, incluyendo las capacidades cognitivas. Estos instrumentos han sido creados con el fin de estudiar a los niños desde el nacimiento y, por lo tanto, para obtener respuestas de sujetos con poca movilidad y lenguaje; además, están diseñados para la realización y valoración de programas de intervención, de manera que se adecuan más que los test de inteligencia clásicos para el estudio de los niños con parálisis cerebral.

El objetivo del presente trabajo es estudiar la evolución durante un año de los niños con parálisis cerebral y retraso mental, analizando los cambios producidos en su desarrollo general y también en las diferentes áreas, incluyendo la adaptativa, personal/social, motora, comunicativa y cognitiva.

\section{Método}

\section{Participantes}

La muestra estuvo compuesta por 41 niños y niñas de entre 1 y 6 años de edad (subgrupos de 1, 2, 3, 4, 5 y 6 años) con el diagnóstico de parálisis cerebral infantil. Los niños eran atendidos en el Servicio de Rehabilitación del hospital Juan Ramón Jiménez, de Huelva. Todos los niños de este grupo habían sufrido complicaciones neurológicas observadas en técnicas de neuroimagen; el $31 \%$ tuvo bajo peso al nacer, el $7 \%$, sufrimiento fetal y un 27 \% sufría epilepsia. Todos los niños de este grupo padecían trastornos motores en algún grado: el 44 \% sufría hemiplejia; el $18 \%$, tetraplejia y el $38 \%$, paraplejia. Los niños estaban divididos en subgrupos de edad (Tabla 1). Todos los niños recibían rehabilitación motora en el hospital y asistían a atención temprana y a clases de apoyo y educación especial en sus respectivos centros escolares.

\section{Procedimiento}

Los sujetos fueron evaluados en dos ocasiones, con un intervalo temporal de 12 meses entre la primera y la segunda evaluación. Este postest se realizo siguiendo el mismo procedimiento y las mismas pruebas que en el pretest. Cada evaluación constaba de una sola sesión. Tras la recogida de datos de la historia clínica y una breve entrevista con los padres o cuidadores, se procedió a aplicar la prueba

\section{TABLA 1}

Medias y desviaciones típicas de las edades en meses de los niños/as que participaron en la investigación. La segunda evaluación se realizó un año después que la primera.

\begin{tabular}{lcccccc}
\hline & \multicolumn{3}{c}{ Pretest } & \multicolumn{3}{c}{ Postest } \\
\hline & $N$ & Media & DE & N & Media & DE \\
\hline 1 año & 9 & 17 & 3.1 & 9 & 30 & 1.2 \\
2 años & 6 & 29 & 3.2 & 6 & 43 & 6.9 \\
3 años & 8 & 40 & 5.6 & 8 & 54 & 4.2 \\
4 años & 7 & 54 & 4.7 & 7 & 63 & 1.3 \\
5 años & 5 & 67 & 3.4 & 5 & 79 & 8.3 \\
6 años & 6 & 75 & 7.3 & 6 & 89 & 3.7 \\
\hline
\end{tabular}

Fuente: elaboración propia. 
de screening del Inventario de Desarrollo de Battelle, según las instrucciones detalladas en el procedimiento de la prueba. Las diferentes subescalas se aplicaron mediante la administración directa al niño, y la entrevista estructurada con los padres o cuidadores para que respondieran a aquellos ítems que no era posible observar en la sala de evaluación (por ejemplo, los relacionados con alimentación, higiene, relación con los iguales, etc.).

\section{Instrumentos}

Se utilizó la adaptación española de la prueba de screening del Inventario de Desarrollo de Battelle (Newbor, Stock \& Wnek, 1996). Esta prueba fue elaborada por un grupo de profesionales de diversos campos para los Laboratorios Columbus del Battelle Memorial Institute. Se desarrolló inicialmente en el año 1984, aunque la adaptación española es de 1996. Es de aplicación individual, está tipificada y se emplea para evaluar las habilidades del desarrollo en niños entre el nacimiento y los ocho años. Proporciona información sobre los puntos fuertes y débiles de los sujetos con el objetivo de elaborar programas individualizados. Se aplica fundamentalmente en el ámbito clínico de la evaluación temprana y la educación especial (Benner, 1992; Culbetson \& Willis, 1993; McLean, Bayley \& Wolery, 1996). Se divide en cinco áreas: personal/social, adaptativa, motora (fina y gruesa), comunicación (receptiva y expresiva) y cognitiva. También ofrece una puntuación total. Los resultados directos se transforman en edades equivalentes de desarrollo. Esta integrado por un total de 341 ítems agrupados en las distintas áreas, y también incluye una prueba de screening formada por 96 ítems, seleccionados entre los que integran el inventario; es precisamente esta prueba la que hemos utilizado en nuestro estudio. Las áreas evaluadas son las siguientes: Área Personal/Social, que estudia la capacidad de realizar interacciones sociales significativas. Área Adaptativa, que evalúa las habilidades de autoayuda. Área Motora, para la capacidad de usar y controlar los músculos del cuerpo (fina y gruesa). Área de Comunicación, que se divide en comunicación receptiva y expresiva. Área Cognitiva, que aprecia habilidades y capacidades de tipo conceptual. El motivo de emplear el screening y no la prueba completa es la mayor adecuación al ámbito clínico, ya que el tiempo requerido para la administración de la segunda hace casi impracticable su utilización en hospitales, mientras que la primera es una opción más breve que permite su aplicación en una sola sesión.

\section{Diseño}

Se trata de una investigación ex post facto de diseño evolutivo secuencial (Montero \& León, 2007), en la que la variable independiente es la maduración de los sujetos y la variable dependiente es la puntuación total y en las diferentes subescalas del screening del Inventario de Desarrollo de Battelle. Las puntuaciones directas fueron transformadas en edades equivalentes utilizando los baremos normativos proporcionados por la prueba. También se transformaron en cocientes de desarrollo, dividiendo las edades equivalentes entre las edades reales y multiplicando el resultado por cien.

\section{Análisis estadístico}

Al tratarse de un diseño ex post facto prospectivo secuencial, se utilizó el test $t$ de Student para muestras independientes en la comparación de variables transversales, y para muestras relacionadas en la comparación de variables longitudinales (Fontes de Gracia et al., 2010). Se empleó el coeficiente de Pearson para estudiar la correlación entre las distintas subescalas. Se consideró significativa una $p<0.05$. Se calcularon los intervalos de confianza al $95 \%$.

\section{Resultados}

Para controlar el posible efecto cohorte, se compararon los sujetos de cada grupo de edad de la primera medición con los de la segunda (es decir, el grupo que tenía dos años en la primera medición con el de la misma edad en la segunda, y así sucesivamente con las distintas edades), sin que se encontrasen diferencias significativas para ningún grupo de edad ( $t$ de Student; todas las $p>0.078$ ). No se en- 


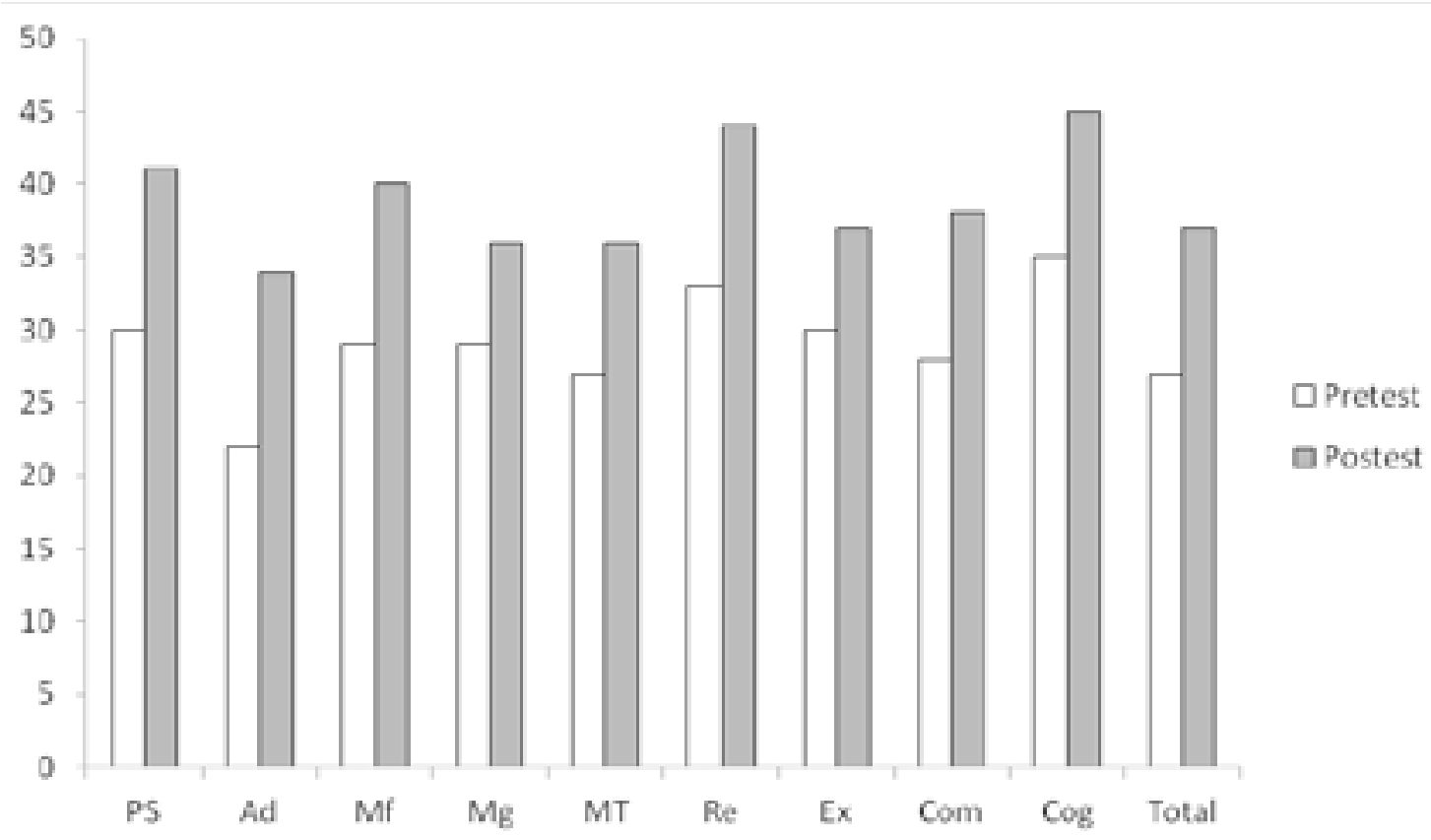

Figura 1. Edades equivalentes en las diferentes subescalas en el pretest y el postest para los sujetos de todos los grupos de edad.

Nota. PS: personal/social; Ad: adaptativa; Mf: motora fina; Mg: motora gruesa; MT: motora total; Re: comunicación receptivas; Ex: comunicación expresiva; Com: comunicación total; Cog: cognitiva.

Fuente: elaboración propia.

contraron diferencias significativas en función del sexo en ninguno de los dos grupos ( $t$ de Student; todas las $p>0.056$ ), de manera que los resultados se muestran agrupados.

Al comparar las edades equivalentes de todos los sujetos obtenidos en la primera medición con los de la segunda (estudio longitudinal), se observó que en la segunda medición obtienen una puntuación significativamente superior en todas las subescalas ( t de Student; todas las $\mathrm{p}<0.007$ ), como se presenta en la Figura 1.

Al comparar la diferencia entre las edades equivalentes de las dos medidas en cada grupo de edad, se encontró que, como se puede apreciar en la

\section{TABLA 2}

Puntuaciones p en la prueba t de Student para muestras relacionadas.

\begin{tabular}{|c|c|c|c|c|c|c|}
\hline Subescalas & $1 \mathrm{a} 2$ & 2 a 3 & 3 a 4 & 4 a 5 & 5 a 6 & 6 a 7 \\
\hline Personal/social & 0.003 & 0.169 & 0.018 & 0.039 & 0.195 & 0.115 \\
\hline Adaptativa & 0.003 & 0.043 & 0.006 & 0.082 & 0.151 & 0.020 \\
\hline Motora fina & 0.017 & 0.095 & 0.107 & 0.137 & 0.100 & 0.534 \\
\hline Motora gruesa & 0.069 & 0.100 & 0.136 & 0.187 & 0.273 & 0.520 \\
\hline Motora total & 0.004 & 0.169 & 0.008 & 0.063 & 0.101 & 0.039 \\
\hline Comunicación receptiva & 0.003 & 0.005 & 0.036 & 0.042 & 0.069 & 0.331 \\
\hline Comuniucación expresiva & 0.053 & 0.042 & 0.561 & 0.001 & 0.294 & 0.179 \\
\hline Comunicación & 0.008 & 0.014 & 0.190 & 0.005 & 0.107 & 0.206 \\
\hline Cognitiva & 0.022 & 0.282 & 0.008 & 0.003 & 0.078 & 0.008 \\
\hline Total & 0.001 & 0.021 & 0.028 & 0.005 & 0.046 & 0.012 \\
\hline
\end{tabular}

Fuente: elaboración propia 


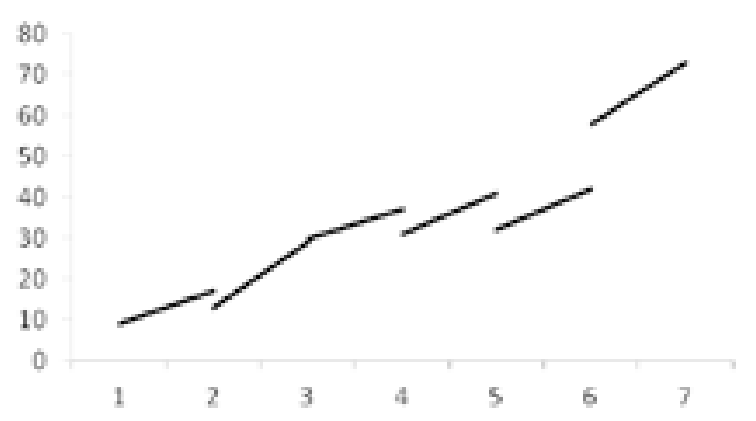

Figura 2. Gráfico de edades equivalentes totales por edad en el pretest y el postes para todos los grupos de edad.

Fuente: elaboración propia.

Tabla 2, los sujetos de todos los grupos obtienen puntuaciones significativamente superiores en el postest realizado un año después, en casi todas las subpruebas del Inventario de Desarrollo de Battelle. En la Figura 2 se presentan las edades equivalentes en el pretest y el postest para la puntuación total.
TABLA 3

Porcentaje de sujetos con cociente de desarrollo menor de 70 (dos desviaciones estándar por debajo de la media) para las diferentes subescalas en el pretest y el postest

\begin{tabular}{lcc}
\hline \multicolumn{1}{c}{ Subescalas } & Pretest & Postest \\
\hline Personal/social & 60 & 52 \\
Adaptativa & 76 & 59 \\
Motora fina & 60 & 47 \\
Motora gruesa & 64 & 61 \\
Motora total & 69 & 59 \\
Comunicación receptiva & 50 & 31 \\
Comuniucación expresiva & 57 & 50 \\
Comunicación & 61 & 50 \\
Cognitiva & 47 & 38 \\
Total & 61 & 52 \\
\hline
\end{tabular}

Fuente: elaboración propia.

Los niños de todos los grupos obtuvieron unas puntuaciones medias inferiores a las esperadas para la muestra normal; por ejemplo, la media de los cocientes de desarrollo totales en el pretest fue de

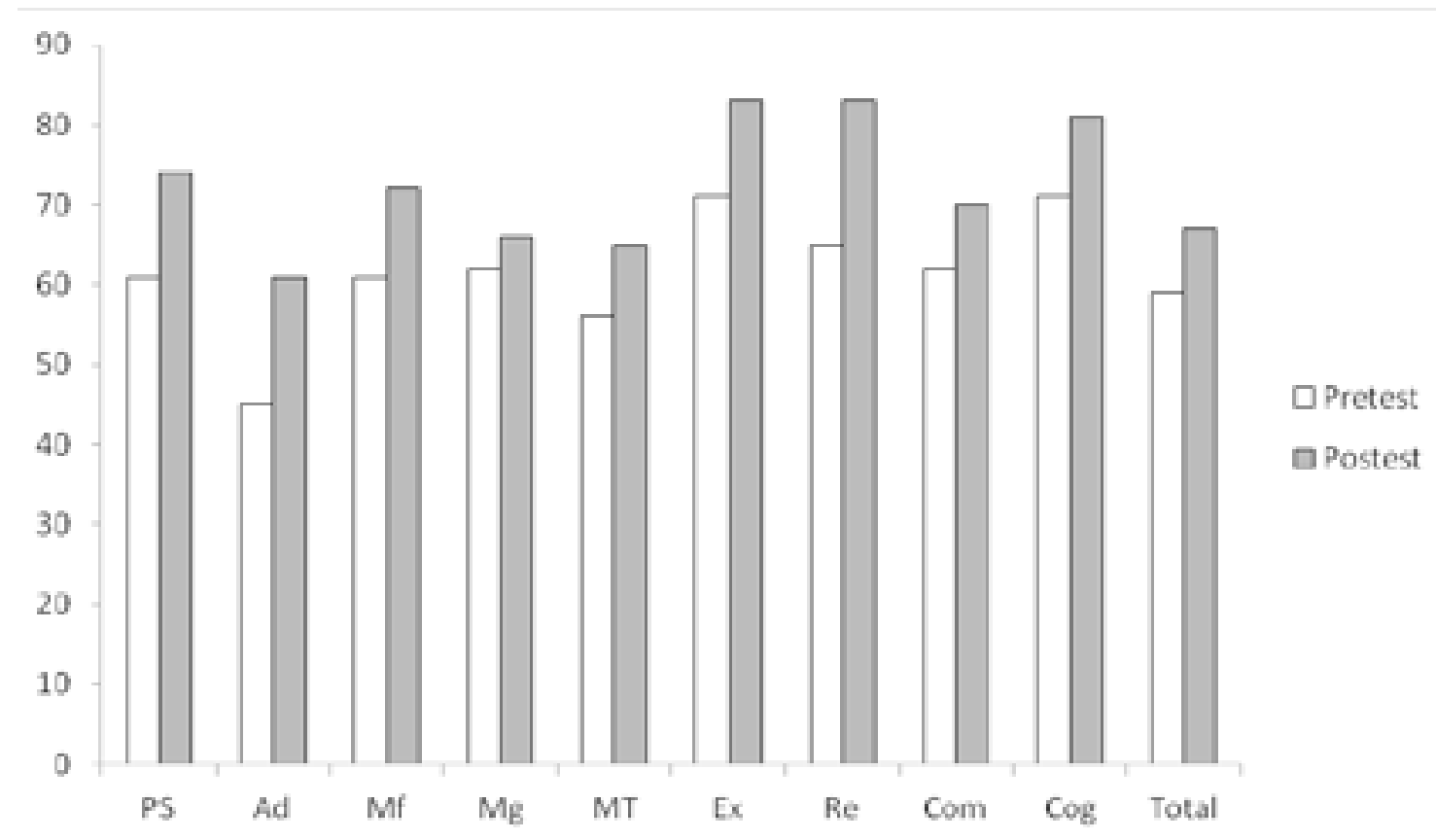

Figura 3. Cocientes de desarrollo en las diferentes subescalas en pretest y el postest para los sujetos de todos los grupos de edad.

Nota. Se encontraron diferencias significativas en todas las subpruebas excepto motora gruesa y comunicación expresiva (PS: personal/social; Ad: adaptativa; Mf: motora fina; Mg: motora gruesa; MT: motora total; Re: comunicación receptivas; Ex: comunicación expresiva; Com: comunicación total; Cog: cognitiva).

Fuente: elaboración propia. 


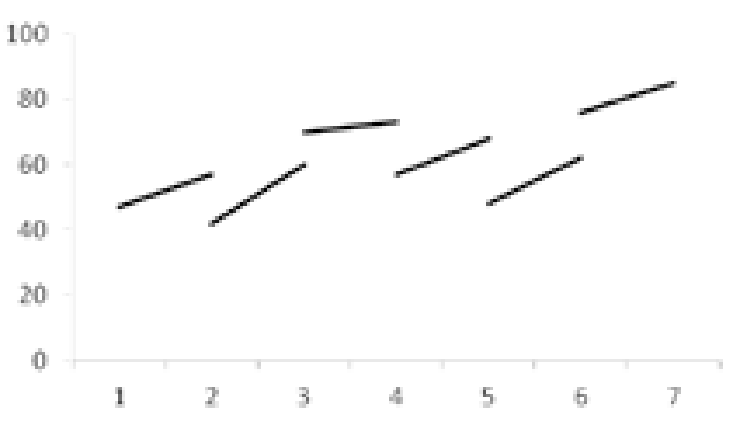

Figura 4. Gráfico de cocientes de desarrollo totales por edad en el pretest y el postest para todos los grupos de edad.

Fuente: elaboración propia.

59 y en el postest de 67 , obteniendo puntuaciones similares en todos los subtest (Figura 3). Un alto porcentaje de niños obtuvo cocientes de desarrollo inferiores a 70 en diferentes áreas evaluadas (Tabla 3), siendo mayor el número de sujetos con puntuaciones inferiores a 70 en el pretest en relación con el postest en todas las subescalas. Por ejemplo, el $47 \%$ de los niños obtuvo en el pretest una puntuación en la escala cognitiva inferior a 70 , mientras que en el postest este porcentaje se redujo al $38 \%$. Lo mismo ocurre en la escala total, pasando de un $61 \%$ a un $52 \%$.

Cuando se comparan los cocientes de desarrollo de los sujetos de todas las edades, se obtienen diferencias significativas en las subescalas personal/social, adaptativa, motora fina, motora total, comunicación receptiva, comunicación total y cognitiva y en la puntuación total (todas las $p<0.031$ ), mientras que no se encuentran diferencias en las subescalas motora gruesa $(p>0.355)$ y comunicación expresiva $(p>0.377)$. Al comparar los sujetos de cada grupo de edad, no se encontraron diferencias en los coeficientes de desarrollo en las diferentes subescalas para ningún grupo de edad (todas las $p>0.052$ ), pero los niños evaluados por primera vez a los 1, 2 y 4 años obtuvieron puntuaciones totales significativamente superiores en la segunda medida realizada un año después (todas las $p<0.013$ ). En la Figura 4 se muestran los cocientes de desarrollo de los diferentes grupos para la puntuación total.

\section{Discusión}

Como cabía esperar, los niños presentaban disfunciones de múltiples áreas de las habilidades cognitivas, lingüísticas, motoras (Damman \& Levinton, 1997) y, por lo tanto, obtuvieron puntuaciones patológicas en la escala de desarrollo (Van Andel et al., 2007). También se cumplieron los pronósticos de la literatura de entre un $30 \%$ y un $70 \%$ de niños con disfunción cognitiva en sujetos con lesión cerebral (Hagberg et al., 1989; Humphreys et al., 2000; Krägeloh-Mann et al., 1995) y que, según la prueba, se encuentra entre un $38 \%$ y un $47 \%$. La comparación entre distintas cohortes no muestra diferencias entre los niños nacidos en un año y los del siguiente.

Los resultados también muestran un aumento generalizado en la edad equivalente en todos los grupos de edad, de modo que aunque los sujetos sufran graves patologías sus puntuaciones mejoran a lo largo del desarrollo, siendo superiores en el postest que en el pretest. Este resultado podría parecer evidente, si no fuera porque los niños no solo mejoran con el tiempo en las edades equivalentes, sino que además lo hacen en los cocientes de desarrollo; es decir, aunque sus puntuaciones continúen siendo patológicas, se muestran menos alejadas de la media en el postest que en el pretest. Otra evidencia de mejora es el número de niños con puntuaciones patológicas, que resulta inferior en el postest que en el pretest (por ejemplo, en la escala total, en la que se pasa de un $61 \%$ a un $52 \%$ de sujetos con puntuaciones de dos desviaciones típicas por debajo de la media). Las dos únicas áreas en las que no se produce esta mejora son la motora gruesa y la de comunicación expresiva, lo cual se evidenció no solo en las puntuaciones obtenidas en la prueba, sino también en las dificultades deambulatorias y del lenguaje oral de gran parte de los sujetos, que se mantuvieron a lo largo del año. Este resultado puede deberse a que la parálisis cerebral es un síndrome que cursa fundamentalmente con trastornos motores. Es decir, la función eferente por excelencia, la motora, es la que se ve más comprometida y es la que ejecuta el habla y la coordinación motora general. 
Resulta difícil explicar el motivo de esta mejora. Pero con todo y según los resultados, es evidente un progreso en el desarrollo, incluso con niños con parálisis cerebral grave, por lo que se podría estar de acuerdo con las explicaciones de que apuntan a la condición dinámica del cerebro, al menos hasta los 6 años de edad. Recordemos que según Bax y Brown (2004), aunque se trata de una condición estática y no progresiva, pueden producirse cambios con el continuo desarrollo del cerebro, por lo que debe considerarse como una condición. No obstante, el aumento generalizado de las puntuaciones podría estar relacionado con los distintos tratamientos e intervenciones que los niños estaban recibiendo, ya que además de la rehabilitación motora en el hospital, todos los niños asistían a clases de apoyo y educación especial en sus respectivos colegios o guarderías, y los más pequeños contaban con la correspondiente atención temprana. No existe unanimidad respecto a la efectividad de este tipo de actuaciones en la mejoría de las capacidades, pues se pueden encontrar tanto estudios que avalan su eficacia (Reddihough, King, Coleman \& Catanese, 1998) como los que la niegan (Jung-Ae, 1997). Para comprobar la influencia real de estos tratamientos en el estudio, hubiese sido necesario utilizar un grupo control de niños que no recibieran ningún tipo de asistencia, dado que afortunadamente esto no sucede en nuestro ámbito, no es viable realizar tal comprobación.

Como limitación de este trabajo, cabe destacar que al tratarse de un estudio ex post facto, resulta imposible controlar todos los factores, como por ejemplo el tipo, calidad e intensidad de la educación especial que recibe cada niño. En este caso concreto, como ya se ha dicho, no es posible buscar un grupo control equivalente que no reciba asistencia. No obstante, el hecho de que se trate de un estudio realizado en un ambiente y condiciones ecológicas, aumenta su validez externa y su extrapolación a otros casos.

Nuestros resultados indican que los niños con parálisis cerebral pueden mejorar significativamente en sus capacidades personales, sociales, adaptativas y cognitivas y en la comunicación receptiva. También apuntan a la posible eficacia de los programas de educación especial y atención temprana en el abordaje de los déficits anteriormente comentados.

\section{Referencias}

American Psychiatric Association. (1994). Diagnostic and statistical manual of mental disorders (4a. ed.). Washington, DC: Autor.

Andersen, G. L., Irgens, L. M., Haagaas, I., Skranes, J. S., Meberg, A. E. \& Vik, T. (2008). Cerebral palsy in Norway: Prevalence, subtypes and severity. European Journal of Paediatric Neurology, 12(1), 4-13.

Bax, M. C. O. (1964). Terminology and classification of cerebral palsy. Developmental Medicine \& Child Neurology, 6(3), 295-297.

Bax, M. \& Brown, J. K. (2004). The spectrum of the motor disorders of children with cerebral palsy. En D. Scrutton, D. Damiano \& M. Mayston (Eds.), Management of the motor disorders of children with cerebral palsy (2 ${ }^{\mathrm{a}}$ ed., pp. 9-21). London: MacKeith Press.

Beckung, E. \& Hagberg, G. (2002). Neuroimpairments, activity limitations, and participation restrictions in children with cerebral palsy. Developmental Medicine \& Child Neurology, 44(5), 309-316.

Benner, S. M. (1992). Assessing young children with special needs: An ecological perspective. New York: Longman.

Cioni, G., Sales, B., Paolicelli, P. B., Petacchi, E., Scusa, M. F. \& Canapicchi, R. (1999). MRI and clinical characteristics of children with hemiplegic cerebral palsy. Neuropediatrics, 30(5), 249-255.

Culbetson, J. L. \& Willis, D. L. (1993). Testing young children: A reference guide for developmental, psychoeducational and psychosocial assessments. Austin, TX: Pro-Ed.

Damman, O. \& Levinton, A. (1997). The role of perinatal brain damage in developmental disabilities: An epidemiologic perspective. Mental Retardation and Developmental Disabililities Research Reviews, 3(1), 13-21.

Fontes de Gracia, S., García-Gallego, C., Quintanilla, L., Rodríguez, R., Rubio, P. \& Sarriá, E. (2010). Fundamentos de investigación en psicología. Madrid: UNED. 
Jung-Ae, J. (1997). Skills for independence for children with cerebral palsy: A comparative longitudinal study. International Journal of Disability, Development and Education, 44(3), 263-274.

Hagberg, B., Hagberg, G. \& Zetterström, R. (1989). Decreasing perinatal mortality, increase in cerebral palsy morbidity? Acta Paediatrica Scandinavica, 78(5), 664-670.

Himmelmann, K., Hagberg, G., Wiklund, L. M., Eek, M. N. \& Uvebrant, P. (2007). Dyskinetic cerebral palsy: A population-based study of children born between 1991 and 1998. Developmental Medicine \& Child Neurology, 49(4), 246-251.

Humphreys, P., Whiting, S. \& Pham, B. (2000). Hemiparetic cerebral palsy: Clinical pattern and imaging in prediction of outcome. The Canadian Journal of Neurological Sciences, 27(3), 210-219.

Krägeloh-Mann, I., Petersen, D., Hagberg, G., Vollmer, B., Hagberg, B. \& Michaelis, R. (1995). Bilateral spastic cerebral palsy - MRI pathology and origin. Analysis from a representative series of 56 cases. Developmental Medicine \& Child Neurology, 37(5), $379-397$.

Krigger, K. W. (2006). Cerebral palsy: An overview. American Family Physician, 73(1), 91-100.

Liptak, G. S. \& Accardo, P. J. (2004). Health and social outcomes of children with cerebral palsy. Journal of Pediatrics, 145(2), 36-41.

McLean, M., Bayley, D. B. \& Wolery, M. (1996). Assessing infants and preschoolers with special needs. Columbus, OH: Charles E. Merrill.

Manuel, J., Naughton, M. J, Balkrishnan, R., PatersonSmith, B. \& Koman, L. A. (2003). Stress and adaptation in mothers of children with cerebral palsy. Journal of Pediatric Psychology, 28(3), 197-201.

Montero, I. \& León, O. G. (2007). A guide for naming research studies in Psychology. International Journal of Clinical and Health Psychology, 7(3), 847-862.

Nieto, M. \& Candau, R. (1995). Parálisis cerebral infantil. En G. Gómez, J. Montilla \& M. Nieto (Eds.), Neurología y neuropsicología pediátrica (pp. 49-80). Jaén: Diputación Provincial de Jaén.
Newbor, J., Stock, J. R. \& Wnek, L. (1996). Inventario de Desarrollo Battelle. Madrid: TEA.

O’Shea, T. M. (2008). Diagnosis, treatment, and prevention of cerebral palsy in Near-Term/Term Infants. Clinical Obstetric and Gynecology, 51(4), 816-828.

Parkes, J., McCullough, N. \& Madden, A. (2010). To what extent do children with cerebral palsy participate in everyday life situations? Health and Social Care in the Community, 18(3), 304-315.

Pueyo, R. \& Vendrell-Gómez, P. (2002). Neuropsicología de la parálisis cerebral. Revista de Neurología, 34(11), 1080-1087.

Puyuelo, M. (1998). Logopedia y parálisis cerebral. En J. Peña (Ed.), Manual de Logopedia (pp. 263-279). Barcelona: Masson.

Puyuelo, M. (2001). Psicología, audición y lenguaje en diferentes cuadros infantiles: aspectos comunicativos y neuropsicológicos. Revista de Neurología, 32(10), 975-980.

Reddihough, D., King, J., Coleman, G. \& Catanese, T. (1998). Efficacy of programs based on conductive education for young children with cerebral palsy. Developmental Medicine \& Child Neurology, 40(11), 763-770.

Surman, G., Newdick, H. \& Johnson, A. (2003). Cerebral palsy rates among low-birthweight infants fell in the 1990s. Developmental Medicine \& Child Neurology, 45(7), 456-462.

Van Handel, M., Swaab, H., de Vries, L. \& Jongmans, M. (2007). Long-term cognitive and behavioral consequences of neonatal encephalopathy following perinatal asphyxia: A review. European Journal of Pediatrics, 166(7), 645-654.

Vargha-Khadem, F., Isaacs, E., Van Der Werf, S., Robb, S. \& Wilson, J. (1992). Development of intelligence and memory in children with hemiplegic cerebral palsy. The deleterious consequences of early seizures. Brain, 115(1), 315-329.

Zafeiriou, D. I., Kontopoulos, E. E. \& Tsikoulas, I. (1999). Characteristics and prognosis of epilepsy in children with cerebral palsy. Journal of Child Neurology, 14(5), 289-294. 
